\title{
BIBECHANA
}

A Multidisciplinary Journal of Science, Technology and Mathematics

ISSN 2091-0762 (online)

Journal homepage: http://nepjol.info/index.php/BIBECHANA

\section{Pseudo-spin Symmetry in Dirac-Four-Parameter Diatomic Problem Coupled with a Coulomb-like Tensor potential}

\author{
Mahdi Eshghi \\ Department of Basic Sciences, Central Tehran Branch, Islamic Azad University, Tehran, Iran \\ e-mail: eshgi54@gmail.com \\ Article history: Received 13 June, 2011; Accepted 21 June, 2011
}

\begin{abstract}
In this work, we use the parametric generalization of the Nikiforov-Uvarov method to obtain the relativistic bound state energy spectrum and the corresponding spinor wave-functions for fourparameter diatomic potential coupled with a Coulomb-like tensor under the condition of the pseudo-spin symmetry. Also, some numerical results have given.
\end{abstract}

Keywords: Dirac equation; four-parameter diatomic potential; Coulomb-like tensor

\section{Introduction}

Since the solutions of the Dirac equation with physical potential are very useful to investigate the relativistic effects, especially a strong coupling system [1]. Recently, some authors have been solved approximately the Dirac equation for some potentials like the Eckart potential [2], ResenMorse potential [3], Poschl-Teller potential [4, 5], Woods-Saxon potential [6], Scarf potential [7, 8], etc. The spin and pseudo-spin symmetry concepts in nuclear theory $[9,10]$, have been used to explain the features of deformed nuclei [11], super-deformation [12], and also to establish an effective nuclear shell-model scheme [13]. Ginocchio has been showed that pseudo-spin symmetry is exact when the sum of the vector potential $V_{v}(r)$ and scalar potential $V_{s}(r)$ is equal to zero or a constant, pseudo-spin symmetry occurs in the Dirac equation [14, 15]. Of course, in real nuclei, $V_{v}(r)+V_{s}(r) \neq$ const. and pseudo-spin symmetry is only an approximation. On the other hands, tensor potentials have been introduced into the Dirac equation with the substitution. In this way, a spin-orbit coupling term was added to the Dirac Hamiltonian [7, 16-23].

The four-parameter diatomic potential (FPDP) [24] following as:

$$
V(r)=-\frac{D}{e^{2 \alpha r}-q}
$$

where $D=D_{e}\left(e^{\varsigma}-1\right), \alpha=\varsigma / r_{e}, D_{e}$ is the depth of the potential well, $r_{e}$ is the equilibrium distance of of the two nuclei, and $\varsigma, q$ are real parameters. 
Recently, C. Gang [25] has been studied solution of the Dirac equation with this Potential by using the supersymmetric quantum mechanics.

The motivation of the present work was to solved the Dirac equation under the pseudo-spin symmetry for FPDP that including a Coulomb-like tensor potential [16]

$$
U(r)=-\frac{H}{r}, \quad H=\frac{Z_{a} Z_{b} e^{2}}{4 \pi \varepsilon_{0}}, \quad r \geq R_{c}
$$

where $R_{c}=7.78 \mathrm{fm}$ is the Coulomb radius, $Z_{a}$ and $Z_{b}$ denote the charges of the projectile $a$ and the target nuclei $b$, respectively.

The parametric generalization of the Nikiforov-Uvarov (NU) method have been used to obtain the Dirac equation with this potential. The energy eigenvalues equation and the corresponding unnormalized eigenfunctions have been obtained.

\section{NU Method}

We give a brief description of the conventional NU method [26]. This method is based on solving the second-order differential equations by means of special orthogonal functions. The mean equation which is closely associated with the method is given in the following form

$$
\psi_{n}^{\prime \prime}(s)+\frac{\tilde{\tau}(s)}{\sigma(s)} \psi_{n}^{\prime}(s)+\frac{\widetilde{\sigma}(s)}{\sigma^{2}(s)} \psi_{n}(s)=0
$$

where $\sigma(s)$ and $\tilde{\sigma}(s)$ are polynomials, at the most of the second degree, and $\tilde{\tau}(s)$ is a polynomials, at most of the first degree. Let us discuss the exact particular solution of Eq. (3) by choosing $\psi_{n}(s)=\phi(s) y_{n}(s)$ resulting in a hypergeometric type equation of the form

$$
\sigma(s) y_{n}^{\prime \prime}(s)+\tau(s) y_{n}^{\prime}(s)+\lambda y_{n}(s)=0
$$

where

$$
\begin{aligned}
& \pi(s)=\sigma(s) \frac{d}{d s}(\ln \varphi(s)) \\
& \tau(s)=\widetilde{\tau}(s)+2 \pi(s), \quad \tau^{\prime}(s)<0
\end{aligned}
$$

where $\pi(r)$ is a polynomial of order at most one.

The first part of the wave function, i.e. $y_{n}(s)$, is the hypergeometric-type function whose polynomials solutions are given by Rodrigue's relation

$$
y_{n}(s)=\frac{a_{n}}{\rho(s)} \frac{d^{n}}{d s^{n}}\left[\sigma^{n}(s) \rho(s)\right]
$$

where $a_{n}$ is a normalization constant and the weight function $\rho(s)$ must satisfy the differential equation

$$
\omega^{\prime}(s)-\left(\frac{\tau(s)}{\sigma(s)}\right) \omega(s)=0, \quad \omega(s)=\sigma(s) \rho(s)
$$

The function $\pi(s)$ and the parameter $\lambda$ in the above equation are defined as follows

$$
\begin{gathered}
\pi(s)=\frac{\sigma^{\prime}(s)-\tilde{\tau}(s)}{2} \pm \sqrt{\left(\frac{\sigma^{\prime}(s)-\tilde{\tau}(s)}{2}\right)^{2}-\tilde{\sigma}(s)+k \sigma(s)} \\
\lambda=k+\pi^{\prime}(s)
\end{gathered}
$$


The determination of $k$ is the essential point in the calculation of $\pi(s)$. It is simply defined by setting the discriminate of the square root which must be zero. The eigenvalues equation have calculated from the above equation

$$
\lambda=\lambda_{n}=-n \tau^{\prime}(s)-\frac{n(n-1)}{2} \sigma^{\prime \prime}(s) . \quad n=0,1,2, \ldots .
$$

In order to clarify the parametric generalization of the NU method, let us take the following equation, which represents a general form of the Schrodinger-like equation written for any potential by an appropriate coordinate transformation $s=s(r)$. Thus, we obtain another generalized hypergeometric equation [18]

$$
\left[s^{2}\left(1-\alpha_{3} s\right)^{2} \frac{d^{2}}{d s^{2}}+s\left(1-\alpha_{3} s\right)\left(\alpha_{1}-\alpha_{2} s\right) \frac{d}{d s}+\left[-\xi_{1} s^{2}+\xi_{2} s-\xi_{3}\right]\right] \psi_{n}(s)=0
$$

When (12) is compared with (3), we get

$$
\tilde{\tau}(s)=\alpha_{1}-\alpha_{2} s, \quad \sigma(s)=s\left(1-\alpha_{3} s\right), \quad \tilde{\sigma}(S)=-\xi_{1} s^{2}+\xi_{2} s-\xi_{3}
$$

Substituting these into (9), we find

$$
\pi(s)=\alpha_{4}+\alpha_{5} s \pm\left[\left(\alpha_{6}-k \alpha_{3}\right) s^{2}+\left(\alpha_{7}+k\right) s+\alpha_{8}\right]^{\frac{1}{2}}
$$

where the parameter set are

$$
\begin{array}{ll}
\alpha_{4}=\frac{1}{2}\left(1-\alpha_{1}\right), & \alpha_{5}=\frac{1}{2}\left(\alpha_{2}-2 \alpha_{3}\right), \quad \alpha_{6}=\alpha_{5}^{2}+\xi_{1}, \\
\alpha_{7}=2 \alpha_{4} \alpha_{5}-\xi_{2}, & \alpha_{8}=\alpha_{4}^{2}+\xi_{3}
\end{array}
$$

We obtain the parameter $k$ from the condition that the function under the square root should be the square of a polynomial

$$
k_{1,2}=-\left(\alpha_{7}+2 \alpha_{3} \alpha_{8}\right) \pm 2 \sqrt{\alpha_{8} \alpha_{9}}
$$

where

$$
\alpha_{9}=\alpha_{3} \alpha_{7}+\alpha_{3}^{2} \alpha_{8}+\alpha_{6}
$$

For each $k$ the following $\pi$ 's are obtained. The function $\pi(s)$ becomes

$$
\pi(s)=\alpha_{4}+\alpha_{5} s-\left[\left(\sqrt{\alpha_{9}}+\alpha_{3} \sqrt{\alpha_{8}}\right) s-\sqrt{\alpha_{8}}\right]
$$

for the $k$-value

$$
k=-\left(\alpha_{7}+2 \alpha_{3} \alpha_{8}\right)-2 \sqrt{\alpha_{8} \alpha_{9}}
$$

We also have from $\tau(s)=\tilde{\tau}(s)+2 \pi(s)$,

$$
\tau(s)=\alpha_{1}+2 \alpha_{4}-\left(\alpha_{2}-2 \alpha_{5}\right) s-2\left[\left(\sqrt{\alpha_{9}}+\alpha_{3} \sqrt{\alpha_{8}}\right) s-\sqrt{\alpha_{8}}\right]
$$

Thus, we impose the following condition to fix the $k$-value

$$
\tau^{\prime}(s)=-\left(\alpha_{2}-2 \alpha_{5}\right)-2\left(\sqrt{\alpha_{9}}+\alpha_{3} \sqrt{\alpha_{8}}\right)=-2 \alpha_{3}-2\left(\sqrt{\alpha_{9}}+\alpha_{3} \sqrt{\alpha_{8}}\right)<0 .
$$

When (10) is used with (20) and (21) the following equation is derived

$$
n\left[(n-1) \alpha_{3}+\alpha_{2}-2 \alpha_{5}\right]+(2 n+1)\left(\sqrt{\alpha_{9}}+\alpha_{3} \sqrt{\alpha_{8}}\right)-\alpha_{5}+\alpha_{7}+2 \alpha_{3} \alpha_{8}+2 \sqrt{\alpha_{8} \alpha_{9}}=0
$$

By using (8)

and together with (7), we have

$$
\rho(s)=s^{\alpha_{10}-1}\left(1-\alpha_{3} s\right)^{\frac{\alpha_{11}-\alpha_{10}-1}{\alpha_{3}}}
$$




$$
y_{n}(s)=P_{n}^{\left(\alpha_{10}-1\right.}, \frac{\left.\alpha_{11}-\alpha_{10}-1\right)}{\alpha_{3}}\left(1-2 \alpha_{3} s\right)
$$

where

$$
\alpha_{10}=\alpha_{1}+2 \alpha_{4}+2 \sqrt{\alpha_{8}}
$$

and

$$
\alpha_{11}=\alpha_{2}-2 \alpha_{5}+2\left(\sqrt{\alpha_{9}}+\alpha_{3} \sqrt{\alpha_{8}}\right)
$$

and $P_{n}^{(\alpha, \beta)}$ are Jacobi polynomials. By using (5), we get

$$
\phi(s)=s^{\alpha_{12}}\left(1-\alpha_{3} s\right)^{-\alpha_{12}-\frac{\alpha_{13}}{\alpha_{3}}}
$$

and the total wave function become

$$
\Psi(s)=s^{\alpha_{12}}\left(1-\alpha_{3} s\right)^{-\alpha_{12}-\frac{\alpha_{13}}{\alpha_{3}}} P_{n}^{\left(\alpha_{10}-1 \quad, \quad \frac{\alpha_{11}-\alpha_{10}-1}{\alpha_{3}}\right)}\left(1-2 \alpha_{3} s\right)
$$

where $\alpha_{12}=\alpha_{4}+\sqrt{\alpha_{8}}$ and $\alpha_{13}=\alpha_{5}-\left(\sqrt{\alpha_{9}}+\alpha_{3} \sqrt{\alpha_{8}}\right)$.

\section{Solution of the Dirac equation}

According to the report which have been given in the researcher $[4,7,16-20]$, the Dirac equation of a nucleon with mass $M$ moving in a scalar and a vector potential including tensor interaction for spin-1/2 particles can be written as $(\hbar=c=1)$,

$$
\left[\vec{\alpha} \cdot \vec{P}+\beta\left(M+V_{s}(r)\right)-i \beta \vec{\alpha} \cdot \hat{r} U(r)\right] \psi_{n k}(\vec{r})=\left[E-V_{v}(r)\right] \psi_{n k}(\vec{r}),
$$

where

$$
\vec{\alpha}=\left(\begin{array}{cc}
0 & \vec{\sigma} \\
\vec{\sigma} & 0
\end{array}\right), \quad \beta=\left(\begin{array}{cc}
I & 0 \\
0 & -I
\end{array}\right)
$$

and $E$ is the relativistic energy of the system and $\vec{P}=-i \vec{\nabla}$ is the three-dimensional momentum operator. In which $\vec{\sigma}$ is vector Pauli matrix. In Pauli-Dirac

$$
\psi_{n k}(\vec{r})=\frac{1}{r}\left[\begin{array}{l}
F_{n k}(r) Y_{j m}^{l}(\theta, \varphi) \\
i G_{n k}(r) Y_{j m}^{\tilde{l}}(\theta, \varphi)
\end{array}\right]
$$

where $F_{n k}(r)$ and $G_{n k}(r)$ are upper- and lower-components of the Dirac spinors and $k$ is eigenvalue of the spin-orbit matrix operator [27]. $Y_{j m}^{l}(\theta, \varphi)$ and $Y_{j m}^{l}(\theta, \varphi)$ are spin pseudospin spherical harmonics, respectively, and $m$ is the projection of the angular momentum on the $z$ axis.

Substituting (31) into (29) and using the following relations [28]

$$
\begin{aligned}
& (\vec{\sigma} \cdot \vec{A})(\vec{\sigma} \cdot \vec{B})=\vec{A} \cdot \vec{B}+i \vec{\sigma} \cdot(\vec{A} \times \vec{B}) \\
& (\vec{\sigma} \cdot \vec{P})=\vec{\sigma} \cdot \hat{r}\left(\hat{r} \cdot \vec{P}+i \frac{\vec{\sigma} \cdot \vec{L}}{r}\right)
\end{aligned}
$$

and properties

$$
\begin{aligned}
& (\vec{\sigma} \cdot \vec{L}) Y_{j m}^{\widetilde{I}}(\theta, \phi)=(k-1) Y_{j m}^{\widetilde{I}}(\theta, \phi) \\
& (\vec{\sigma} \cdot \vec{L}) Y_{j m}^{l}(\theta, \phi)=-(k-1) Y_{j m}^{l}(\theta, \phi)
\end{aligned}
$$


Mahdi Eshghi / BIBECHANA 8 (2012) 23-30 : BMHSS, p. 27

$$
\begin{aligned}
& (\vec{\sigma} . \hat{r}) Y_{j m}^{\tilde{l}}(\theta, \phi)=-Y_{j m}^{l}(\theta, \phi) \\
& (\vec{\sigma} . \hat{r}) Y_{j m}^{l}(\theta, \phi)=-Y_{j m}^{\widetilde{l}}(\theta, \phi)
\end{aligned}
$$

yields two coupled differential equations as follows

$$
\begin{aligned}
& \left(\frac{d}{d r}+\frac{k}{r}-U(r)\right) F_{n k}(r)=\left[E_{n k}+M-\Delta(r)\right] G_{n k}(r) \\
& \left(\frac{d}{d r}-\frac{k}{r}+U(r)\right) G_{n k}(r)=\left[M-E_{n k}+\Sigma(r)\right] F_{n k}(r)
\end{aligned}
$$

where $\Delta$ and $\Sigma$ have been assumed to be radial functions, i.e., $\Delta(r)=V_{v}(r)-V_{s}(r)$ and $\Sigma(r)=V_{v}(r)+V_{s}(r)$.

By substituting $G_{n k}(r)$ from (38) into (39) and $F_{n k}(r)$ from (39) into (38), we have been obtained the following two second-order differential equations for the upper and lower components,

$$
\begin{aligned}
& \left\{\frac{d^{2}}{d r^{2}}-\frac{k(k+1)}{r^{2}}+\frac{2 k}{r} U(r)-\frac{d U(r)}{d r}-U^{2}(r)\right. \\
& \left.+\left(E_{n k}+M-\Delta(r)\right)\left(E_{n k}-M-\Sigma(r)\right)+\frac{-\frac{d \Delta(r)}{d r}}{\left(M+E_{n k}-\Delta(r)\right)}\left(\frac{d}{d r}+\frac{k}{r}\right)\right\} F_{n k}(r)=0 \\
& \left\{\frac{d^{2}}{d r^{2}}-\frac{k(k-1)}{r^{2}}+\frac{2 k}{r} U(r)+\frac{d U(r)}{d r}-U^{2}(r)\right. \\
& \left.+\left(E_{n k}+M-\Delta(r)\right)\left(E_{n k}-M-\Sigma(r)\right)+\frac{\frac{d \Sigma(r)}{d r}}{\left(M-E_{n k}+\Sigma(r)\right)}\left(\frac{d}{d r}-\frac{k}{r}\right)\right\} G_{n k}(r)=0
\end{aligned}
$$

In the above equations $k(k+1)=l(l+1)$ and $k(k-1)=\tilde{l}(\tilde{l}+1)$.

Substituting (1) and (2) into (41), considering pseudo-spin symmetry, taking $\Delta(r)$ as the FPDP and $\Sigma(r)=C_{p s}=$ const. $(d \Sigma(r) / d r=0)$, [29, 30] i.e., the equation have been obtained for the upper component of the Dirac spinor $F_{n k}(r)$ becomes

$$
\begin{aligned}
\left\{\frac{d^{2}}{d r^{2}}-\right. & \frac{(k+H)(k+H-1)}{r^{2}} \\
& \left.+\left(M+E_{n k}\right)\left(M+E_{n k}-C_{p s}\right)-\left(E_{n k}-M-C_{p s}\right)\left[-\frac{D}{e^{2 \alpha r}-q}\right]\right\} F_{n k}(r)=0
\end{aligned}
$$

This equation is describes a particle of spin-1/2 such as the electron in the Dirac theory with FPDP potential including a tensor coupling, that can not be solved analytically because of $(k+H)(k+H-1) / r^{2}$ term, we take the following approximation [31, 32]

$$
\frac{1}{r^{2}} \approx \frac{1}{r_{0}^{2}}\left[C_{0}+\frac{C_{1}}{e^{\alpha r}-q}+\frac{C_{2}}{\left(e^{\alpha r}-q\right)^{2}}\right]
$$

where $C_{0}, C_{1}$ and $C_{2}$ are real constant, $r_{0}=\frac{1}{\alpha} \cdot \log \left[1+2 \alpha^{\prime}\left(\alpha^{\prime}-1\right) / A\right]$, where $\alpha, \alpha^{\prime}$ and $A$ are constant and $q$ is real parameter and constant, also [31]. By using a transformation of the form $s=e^{\alpha r}$, we rewrite it as follows 
where

$$
\left.\frac{d^{2}}{d s^{2}}+\frac{s-q}{s(s-q)} \frac{d}{d s}+\frac{1}{[s(s-q)]^{2}}\left[\xi_{1} s^{2}+\xi_{2} s+\xi_{3}\right]\right\} F_{n k}(s)=0
$$

$$
\begin{aligned}
& \xi_{1}=\frac{C_{0}(k+H)(k+H-1)}{r_{0}^{2} \alpha^{2}}-\frac{\left(E_{n k}-M-C_{p s}\right)\left(E_{n k}+M\right)}{\alpha^{2}}, \\
& \xi_{2}=\left[\frac{(k+H)(k+H-1)\left(2 q C_{0}-C_{1}\right)}{r_{0}^{2} \alpha^{2}}-\frac{\left(E_{n k}-M-C_{p s}\right)\left[2 q\left(E_{n k}+M\right)-D\right]}{\alpha^{2}}\right], \\
& \xi_{3}=\frac{(k+H)(k+H-1)\left(q^{2} C_{0}-C_{1} q+C_{2}\right)}{r_{0}^{2} \alpha^{2}}-\frac{\left(E_{n k}-M-C_{p s}\right)\left[(E+M) q^{2}-D q\right]}{\alpha^{2}}
\end{aligned}
$$

By comparing (44) with (12), we have been obtained the parameter set as

$$
\begin{aligned}
& \alpha_{1}=-q, \quad \alpha_{2}=1, \quad \alpha_{3}=1, \quad \alpha_{4}=(1+q) / 2, \quad \alpha_{5}=-1 / 2, \quad \alpha_{6}=1 / 4+\xi_{1}, \\
& \alpha_{7}=-(1+q) / 2-\xi_{2}, \quad \alpha_{8}=(1+q)^{2} / 4+\xi_{3}, \quad \alpha_{9}=\xi_{1}-\xi_{2}+\xi_{3}+q^{2} / 4, \\
& \alpha_{10}=1+2 \sqrt{(1+q)^{2} / 4+\xi_{3}}, \quad \alpha_{11}=2+2\left(\sqrt{\xi_{1}-\xi_{2}+\xi_{3}+q^{2} / 4}+\sqrt{(1+q)^{2} / 4+\xi_{3}}\right), \\
& \alpha_{13}=-\frac{1}{2}-\left(\sqrt{\xi_{1}-\xi_{2}+\xi_{3}+q^{2} / 4}+\sqrt{(1+q)^{2} / 4+\xi_{3}}\right)
\end{aligned}
$$

The energy eigenvalue function is obtained from eq. (22) as follows:

$$
\begin{aligned}
n[(n-1) & +2]+(2 n+1)\left(\sqrt{\xi_{1}-\xi_{2}+\xi_{3}+q^{2} / 4}+\sqrt{(1+q)^{2} / 4+\xi_{3}}\right) \\
& +1 / 2-(1+q) / 2-\xi_{2}+2\left((1+q)^{2} / 4+\xi_{3}\right)+2 \sqrt{\left(\xi_{1}-\xi_{2}+\xi_{3}+q^{2} / 4\right)\left((1+q)^{2} / 4+\xi_{3}\right)}=0 \text { (47) }
\end{aligned}
$$

Some numerical results are given in table 1. we use the parameters $C_{p s}=-6, \quad M=1$, $\alpha=0.05, C_{0}=0.000208178, C_{1}=0.002500011408, C_{2}=0.002499999716, \quad A=30.52, \quad \alpha^{\prime}=1.5$, $H=5, q=1, D=-0.03815$. The lower component of the Dirac spinor is obtained from (28) as follows:

$$
\begin{aligned}
& G_{n k}(s)=B_{n k} s^{\frac{(1+q)}{2}+\sqrt{(1+q)^{2} / 4+\xi_{3}}}(1+s)^{-\frac{q}{2}+\sqrt{\xi_{1}-\xi_{2}+\xi_{3}+q^{2} / 4}}
\end{aligned}
$$

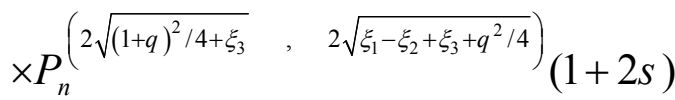

where $B_{n k}$ is the normalization constant to be determined from the normalization condition

$$
\int_{0}^{\infty}\left|G_{n k}(r)\right|^{2} d r=1=b \int_{0}^{1} s^{-1}\left|G_{n k}(s)\right|^{2} d s
$$

and

$$
\begin{array}{r}
\xi_{1}-\xi_{2}+\xi_{3}=\frac{(k+H)(k+H-1)\left[C_{0}(1-q)^{2}+C_{1}(1-q)+C_{2}\right]}{r_{0}^{2} \alpha^{2}} \\
-\frac{\left(E_{n k}-M-C_{p s}\right)\left[(M+E)(1-q)^{2}+D(1-q)\right]}{\alpha^{2}}
\end{array}
$$


Table 1. The bound state energy eigenvalues $E_{n, k}$ in unit of $\mathrm{fm}^{-1}$ of the pseudospin symmetry FPDF for several values of $n$ and $k$.

\begin{tabular}{ccccccccc}
\hline$\tilde{l}$ & $n, k<0$ & $l, j$ & $E_{n, k<0}(H=0)$ & $E_{n, k<0}(H=5)$ & $n-1, k>0$ & $l+2, j+1$ & $E_{n-1, k>0}(H=0)$ & $E_{n-1, k>0}(H=5)$ \\
\hline 1 & $1,-1$ & $2 s_{1 / 2}$ & -4.394273445 & -3.665027110 & 0,2 & $4 d_{3 / 2}$ & -4.394273445 & -2.753155376 \\
2 & $1,-2$ & $3 p_{3 / 2}$ & -4.031394416 & -4.031394416 & 0,3 & $5 f_{5 / 2}$ & -4.031394416 & -2.528115228 \\
3 & $1,-3$ & $4 d_{5 / 2}$ & -3.665027110 & -4.394273445 & 0,4 & $6 g_{7 / 2}$ & -3.665027110 & -2.338132323 \\
4 & $1,-4$ & $5 f_{7 / 2}$ & -3.322587859 & -4.712300356 & 0,5 & $7 h_{9 / 2}$ & -3.322587859 & -2.178334872 \\
\hline
\end{tabular}

\section{Conclusion}

In this paper, by using the parametric generalization of the NU method, we have been solved analytically the approximate energy eigenvalues equation and the corresponding unnormalized wave-functions of the Dirac equation for the FPDP coupled with a Coulomb-like tensor under the condition of the pseudo-spin symmetry. Some numerical results are given for this potential.

\section{Acknowledgments}

The author would like to thank the kind referee for their positive suggestions which have improved the present work.

\section{References}

[1] I.C. Wang and C.Y. Wong, Phys. Rev. D 38 (1988) 348.

[2] X. Zou, L.Z. Yi and C.S. Jia, Phys. Lett. A 346 (2005) 54.

[3] W.C. Qiang, R.S. Zhou and Y. Gao, J. Phys. A: Math. Theor. 40 (2007) 1677.

[4] M. Eshghi and H. Mehraban, Few-Body Syst. (2011), doi:10.1007/s00601-011-0238-5.

[5] M. Eshghi and H. Mehraban, Europ. J. Sci. Res. 54 (2011) 22.

[6] Q. Xu and S.J. Zhu, Nucl. Phys. A 768 (2006) 161.

[7] M. Eshghi and H. Mehraban, J. Sci. Res. 3 (2011) 239.

[8] H. Motavali, Mod. Phys. Lett. A 24 (2009) 1227.

[9] A. Arima, M. Harvey and K. Shimizu, Phys. Lett. B 30 (1969) 517.

[10] K.T. Hecht and A. Adler, Nucl. Phys. A 137 (1969) 129.

[11] A. Bohr, I. Hamamoto and B.R. Mottelson, Phys. Scr. 26 (1982) 267.

[12] J. Dudek, W. Nazarewicz, Z. Szymanki and G.A. Leander, Phys. Rev. Lett. 59 (1987) 1405.

[13] D. Troltenier, C. Bahri and J.P. Draayer, Nucl. Phys. A 586 (1995) 53.

[14] J.N. Ginocchio, Phys. Rev. Lett. 78 (1997) 436.

[15] J.N. Ginocchio, Phys. Rep. 315 (1999) 231.

[16] M. Hamzavi, A.A. Rajabi and H. Hassanabadi, Phys. Lett. A 374 (2010) 4303.

[17] H. Akcay and C. Tezcan, Int. J. Mod. Phys. C 20 (2009) 931.

[18] M. Hamzavi, H. Hassanabadi and A.A. Rajabi, Int. J. Theor. Phys. 50 (2010) 454.

[19] S. Zarrinkamar, A.A. Rajabi and H. Hassanabadi, Ann. Phys. 325 (2010) 2522.

[20] H. Akcay, Phys. Lett. A 373 (2009) 616.

[21] O. Aydogdu and R. Sever, Few-Boby Syst. 47 (2010) 193.

[22] M. Hamzavi, A.A. Rajabi and H Hassanabadi, Few-Body Syst. 48 (2010) 171.

[23] M. Hamzavi, A.A. Rajabi and H. Hassanabadi, Int. J. Mod. Phys. A 26 (2011) 1363. 
[24] J.-X. sun, Acta Phys. Sin. 48 (1999) 1992.

[25] C. Gang, Phys. Lett. A 328 (2004) 116.

[26] A.F. Nikiforov and V.B. Uvarov, Special Functions of Mathemathical Physics (Basel: Birkhauser)1988.

[27] C.Y. Chen, Phys. Lett. A 339 (2005) 283.

[28] J.D. Bjorken and S.D. Drell, Relativistic Quantum Mechanics (McGraw-Hill, NY) 1964.

[29] J. Meng, K. Sugawara-Tanaha, S. Yamaji and A. Arima, Phys. Rev. C 59 (1999) 154.

[30] J. Meng, K. Sugawara-Tanaha, S. Yamaji, P. Ring and A. Arima, Phys. Rev. C 58 (1998) R628.

[31] G.F. Wei and S.H. Dong, Phys. Lett. B 686 (2010) 288.

[32] S.M. Ikhdair and R. Sever, Appl. Math. Com., 216 (2010) 911. arXiv:1001.4327. 